\title{
Primary adenocarcinoma of the appendix
}

\author{
N. K. MENON \\ M.B., F.R.C.S. \\ Department of Surgery, St Margaret's Hospital, Epping, Essex
}

\begin{abstract}
Summary
Two cases of carcinoma of the appendix presenting as appendicular masses are discussed. Carcinoma of the appendix is rare and hence a pre-operative diagnosis is seldom made. Awareness of the condition would naturally arouse suspicion of its presence, especially in elderly patients who present with acute appendicitis or an appendix mass. It is known to have occurred in an appendix stump several years after the initial appendicectomy, and also in a 17-year-old patient. The need for histological examination of all appendicetomy specimens is stressed. The operation of choice is right hemicolectomy, either as a primary or as a secondary procedure. This is associated with better survival rates than when the condition is treated by appendicectomy alone.
\end{abstract}

\section{Case reports}

Case 1

A 77-year-old man was admitted with a 3-week history of continuous pain in the right iliac fossa of moderate severity. He was otherwise asymptomatic with a normal appetite and bowel action. He had not been vomiting.

On examination he looked well, was afebrile and not in obvious pain. The abdomen was not distended and moved normally with respiration. He was tender in the right iliac fossa with mild guarding over a mass therein which was $10 \mathrm{~cm}$ in diameter and firm in character. Rectal examination was unremarkable. His WCC was $17.9 \times 10^{9} / 1$ with a differential count of $82 \%$ neutrophils, $14 \%$ lymphocytes and $4 \%$ monocytes.

On a clinical diagnosis of an appendicular mass he was managed conservatively and appeared to recover with gradual resolution of the mass. But he suddenly collapsed and died on the 13th day after admission. Post-mortem revealed multiple small pulmonary emboli with a large embolus in the right lower lobe pulmonary artery. The clinical signs in the right iliac fossa were due to an inflammatory mass with local peritonitis. There was a primary carcinoma of the base of the appendix which was occluding its lumen. Distal to this the appendix was inflamed with perforation adjacent to the carcinoma. The regionalo lymph nodes were not enlarged and the liver wase free of metastases.

\section{Case 2}

A 65 -year-old epileptic patient was on a routine_evaluation at the neurology clinic when, in passing, $\subset$ he complained of a dull ache in the right iliac fossa of one month's duration. He was otherwise asympto- $-\overrightarrow{0}$ matic and in good health.

Abdominal examination revealed a $10-\mathrm{cm}$ di meter mass in the right iliac fossa over which he vẩs mildly tender with minimal guarding. No others abnormality was detected. His WCC was $15.4 \times 10^{9} / 10$ with a differential count of $83 \%$ neutrophils, $12 \%$ lymphocytes and $5 \%$ monocytes. Barium enemå examination showed the contrast to run freely into the caecum where it stopped short and failed to enter 3 either the terminal ileum or the appendix. No gas shadows were seen in the right iliac fossa and thereo was an upward curve on the column of barium but there did not appear to be a definite tumour in the wall of the caecum. The appearances were nonspecific and were thought to be due to an appendic 3 . ular abscess, a caecal neoplasm or due to externalô involvement by a tumour. He was admitted for surgery a fortnight later when on examination it was? found that the mass had virtually disappeared leaving only a smaller but distinct lump in the righ? iliac fossa.

Subsequent exploration at surgery showed a mass involving the caecum and the base of the appendix with a soft distal part of the appendix containing pus. The regional lymph nodes were not involved by tumour and the liver was free of metastases. Ono

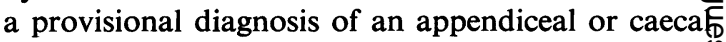
neoplasm a right hemicolectomy was performed $\stackrel{\mathcal{N}}{+}$ He made an uneventful recovery postoperatively ando remains (1979) in good health.

The macroscopic features of the resected specimer 
consisted of a red appendix which on section appeared diffusely infiltrated by a tumour. Microscopic examination revealed invasion of the appendix by a colloid adenocarcinoma which had extended locally through the wall of the viscus. Sections of the mesenteric lymph showed them to be free of tumour involvement.

\section{Discussion}

The first case of carcinoma of the appendix was described by Berger in 1882 . The number of similar cases described in the English literature is fewer than 200 to date. It constitutes $0.2 \%-1.0 \%$ of all intestinal malignant tumours (Didolkar and Fanous, 1977) while the incidence of adenocarcinoma in appendicectomy specimens has been reported as ranging from $1 / 700$ to $1 / 1600$ (Collins, 1955). Carcinoid of the appendix occurs 10 times more commonly than primary adenocarcinoma (Lesnick and Miller, 1949). The peak incidence is in the 6th and 7 th decades and $90 \%$ of cases occur in patients over the age of 40 years. The youngest reported case was in a 17-year-old patient (Pugeda and Hinshaw, 1969). Males are affected more commonly than females (Gamble, 1976; Didolkar and Fanous, 1977).

Tumours of the appendix are of 3 histological types: 1 , carcinoid, $88 \%$; 2 , cystic adenocarcinoma or malignant mucocoele, $8 \%$; and 3 , adenocarcinoma, 4\% (Ulhein and McDonald, 1943). Guarino and Chitwood (1954) defined 2 criteria which they claimed needed to be satisfied before a diagnosis of appendicular carcinoma could be made, namely (i) that there should be continuity between the carcinoma and the mucosa of the appendix; (ii) the presence of mucin-containing neoplastic acini within the tumour, to exclude it from a simple mucocoele of the appendix.

Wolff and Ahmed (1976) used the following criteria to assist in their diagnosis of the condition: (a) when both the caecum and the appendix are involved in continuity by the tumour, only those cases where the major part of the tumour is in the appendix are considered; (b) when the tumour involves the serosa and the likelihood of intraperitoneal spread of another tumour arises, the relative amounts of tumour in the various layers of the appendix are considered. If the greater part of the tumour is near the mucosa and only a minor part reached the serosa, then the tumour is considered to be of appendiceal origin.

Carcinoma of the appendix may take the form of a well differentiated papillary adenocarcinoma which may produce pseudomyxoma peritonii, or that of a solid undifferentiated tumour. In between these 2 extremes, varying degrees of differentiation may occur. The solid tumours usually produce little mucus and resemble colonic adenocarcinoma
(Gamble, 1976). Wolff and Ahmed (1976) had a 50\% incidence, in their series, of poorly differentiated tumours consisting of signet-ring cells forming microglandular structures. This is a high incidence, since in the rest of the colon only 60 cases of this type of tumour have been noted (Wolff and Ahmed, 1976). Spread of the tumour is by direct extension to and local invasion of adjacent structures, and also by the lymphatics and blood. At one or 2 points in the appendix the muscular layers are frequently incomplete or absent (Warwick and Williams, 1973), which explains why direct extension of carcinoma of the appendix may occur early (Gamble, 1976). Invasion of the urinary bladder (Richie and Smith, 1977) and involvement of the right ureter (Aburahma, 1977) have been described. Lymphatic spread is initially to the ileo-colic nodes and later to the infraduodenal and para-aortic nodes (Otto et al., 1970). Liver metastases are common (Aburahma, 1977). Peritoneal implants of tumour are frequent and are probably related to rupture of the appendix (Davis, 1975). Pseudomyxoma peritonei and venous thrombo-embolism due to carcinoma of the appendix have been reported by Williams (1975).

Symptoms are due to obstruction of the lumen of the appendix by the tumour (Berman and James, 1970), infiltration by tumour into the wall of the appendix with or without perforation or by obstruction of the lymphatics or vasculature of the appendix (Nicebert, Feldman and Mandeberg, 1956). Intussusception of the appendix into the lumen of the caecum may also occur (Gamble, 1976). Hesketh (1963) reported 94 cases of adenocarcinoma of the appendix of which $44 \%$ presented as acute appendicitis, $14 \%$ as appendicular abscess, $11 \%$ as chronic appendicitis, $11 \%$ terminally, and $14 \%$ of the cases were detected at incidental laparotomy for an unrelated intra-abdominal procedure. Six per cent. of the cases presented initially as metastases. A chronic inflammatory mass in the right iliac fossa was reported by Didolkar and Fanous (1977) to be a rare manifestation of the condition. Nevertheless, the 2 cases described in this paper presented in this manner. In case 2 the initially large mass in the right iliac fossa resolved leaving a small non-tender mass in the same region. He was relatively asymtomatic during the entire course of his illness and barium enema examination suggested a caecal neoplasm. This case illustrates the need for radiological investigation of the large bowel in patients after middle age whose appendicular masses have resolved, and before exploration. In the reports of Coblentz and Filippone (1975) and Schmutzer, Bayar and Zaki (1975) carcinoma of the appendix was an incidental finding at laparotomy and Forsgren, Molin and Rieger (1974) stressed the importance of palpating the appendix at every laparotomy 
and the need for histological examination of all appendices that are removed at surgery.

It is difficult to recognize a carcinoma in an acutely inflamed appendix. It is therefore important that every appendix removed should be slit open and carefully examined. Didolkar and Fanous (1977) recommended frozen section examination of the tumour to enable diagnosis in such cases, and right hemicolectomy as the definitive treatment if the condition of the patient and that of the bowel permit it. Most writers are agreed that right hemicolectomy is the treatment of choice for carcinoma of the appendix, although Sieracki and Tesluk (1956) recommended appendicectomy only if the tumour is confined to the mucosa. However, owing to the peculiar anatomy of the appendix, where at one or 2 points the mucosa and the submucosa are in close apposition to the serosa, a sub-mucosal tumour is in fact sub-serosal. For this reason Pugeda and Hinshaw (1969) advise right hemicolectomy as being the ideal treatment. Furthermore, McCollum and Pund (1951) found a marked similarity between the cells of pre-invasive carcinoma of the appendix and those of the invasive stage, and DasGupta and Paglia (1966) consider appendicectomy alone unsatisfactory in the non-invasive stage because it is impossible to predict the malignant potential of an individual appendicular carcinoma. If histological examination after appendicectomy alone reveals carcinoma, then right hemicolectomy should be performed at the earliest possible instance (Didolkar and Fanous, 1977). Hopkins, Tullis and Kristensen (1973) showed a significant increase in survival of patients treated by right hemicolectomy as compared to those treated by appendicectomy alone, and Hesketh (1963) reported the 5-year survival rates after these 2 procedures to be $63 \%$ and $20 \%$ respectively. Brown and Husni (1957) advised against radical resection in ruptured appendiceal carcinoma although Edmondson and Hobbs (1967) and Didolkar and Fanous (1977) showed that right hemicolectomy gives good results even in such cases.

\section{Acknowledgments}

My thanks are due to Mr H. W. A. Baron, Senior Consultant Surgeon, St Margaret's Hospital, Epping, under whose care these patients were managed, for permission to publish these cases.

\section{References}

Aburahma, A.F. (1977) Primary adenocarcinoma of the vermiform appendix: report of five new cases and review of the literature. West Virginia Medical Journal, 73, 296.

Berger, A. (1882) Ein Fall von Krebs des Wurm-fortsatzes. Berliner klinischer Wochenschrift, 19, 616.
Berman, A.T. \& JAMES Jr, P.M. (1970) Adenocarcinoma o̊ the appendix. American Journal of Surgery, 119. 733.

BRown, H.W. \& HUSNI, E.A. (1957) Ruptured adenocar $\stackrel{\mathbb{D}}{2}$ cinoma of the appendix: review of the literature and case presentations. Surgery, 42, 953.

Coblentz, M.G. \& Filippone, D.R. (1975) Primary adeno carcinoma of the appendix. Surgery, 77, 160.

Collins, D.C. (1955) A study of 50,000 specimens of the human vermiform appendix. Surgery, Gynecology and Obstetrics, 101, 437.

DasGupta, J.K. \& Paglia, M.A. (1966) Primary malignanid tumors of the appendix. New York Medical Journal, 66

Davis, J. (1975) Primary invasive adenocarcinoma of theappendix. Nebraska Medical Journal, 60, 4.

DidolkaR, M.S. \& FANOus, N. (1977) Adenocarcinoma of the appendix: a clinicopathological study. Diseases of the Colon and Rectum, 20, 130.

Edmondson Jr, H.J. \& HobBs, M.L. (1967) Primary adeno@ carcinoma of the appendix. American Surgeon, 33, 717. 3 .

Forsgren, L. Molin, K. \& Rieger, A. (1974) Adenocarcin-cr oma of the vermiform appendix. Acta chirurgica scandin-? avica, 140, 486.

GAMBLE, H.A. II (1976) Adenocarcinoma of the appendix:の̆ an unusual case and review. Diseases of the Colon and Rectum, 19, 621.

Guarino, G.B. \& Chitwood Jr, E.M. (1954) Adenocarcin-o oma of the appendix: with a review of recent literature. American Journal of Surgery, 87, 293.

HeSKeTH, K.T. (1963) The management of primary adenocarcinoma of the vermiform appendix. Gut, 4, 158.

Hopkins, G.B., Tullis, R.H. \& KRISTEnSEN, K.A.B. (1973) Primary adenocarcinoma of the appendix: report of sergn cases and review of the literature. Diseases of the Coton and Rectum, 16, 140.

LeSNiCK, G. \& Miller, D. (1949) Adenocarcinoma of appendix. Cancer, 2, 18.

McCollum, W. \& Pund, E.R. (1951) Pre-invasive adenocarcinoma of the appendix: report of sixteen cases. Cancer. New York, Philadelphia, etc., 4, 261.

Nicebert, D.M., Feldman, S. \& Mandeberg, A. (1956)』 Adenocarcinoma of the vermiform appendix: a case report $\overrightarrow{\vec{B}}$ and review of the literature. Surgery, 40, 560.

Otro, R.E., Ghislandi, E.V., LoRenzo, G.A. \& Conn Jr, J. (1970) Primary appendiceal carcinoma. American Journal of Surgery, 120, 706.

Pugeda, F.V. \& Hinshaw, J.R. (1969) Primary adenocarcinoma of the appendix. Diseases of the Colon and Rectum, $12,457$.

RichIE, J.P. \& SMITH, A. (1977) Primary adenocarcinoma of $\frac{0}{3}$ the appendix. Archives of Surgery, 112, 666.

SCHMUTZER, K.J., BAYAR, M. \& ZAKI, A.E. (1975) Tumourso of the appendix. Diseases of the Colon and Rectum, 18, 324.

SieraCKI, J.C. \& Tesluk, H. (1956) Primary adenocarcinoma 을 of the vermiform appendix. Cancer. New York, Phila- $D$ delphia, etc., 9, 997.

Ulhein, A. \& MCDonald, J.R. (1943) Primary carcinoma of the appendix resembling carcinoma of the colon. Surgery, $\sigma$ Gynecology and Obstetrics, 76, 711. WARWICK, R. \& WiLliams, P.L. (Eds) (1973) Gray's Anatomy,

Williams, R.W. (1975) Carcinoma of the appendix with pseudomyxoma peritonei and venous thromboembolism. British Journal of Clinical Practice, 29, 19.

WolfF, M. \& AHMED, N. (1976) Epithelial neoplasma of the vermiform appendix (exclusive of carcinoid): I. Adeno-? carcainoma of the appendix. Cancer. New York, Phila- $\square$ delphia, etc., 37, 2493. 